\title{
Triethyl Benzyl Ammonium Chloride (TEBAC) Catalyzed Solvent Free Synthesis of Polyhydroquinolines:A Green Chemistry Aspect
}

\author{
VIJENDER GOEL, ANJU BAJWAN, SONU CHAUHAN and SHARDA GOEL* \\ Department of Chemistry, Maharshi Dayanand University, Rohtak-124001, Haryana, India \\ vkg108@gmail.com
}

Received 17 November 2017 / Accepted 4 December 2017

\begin{abstract}
An environmentally benign, efficient and convenient protocol has been developed for the synthesis of derivatives of polyhydroquinolines by combining dimedone, ethylacetoacetate and ammonium acetate with various substituted arylaldehydes in excellent yields by a grinding method under TEBAC catalyzed solvent-free conditions. The process is simple, straightforward, environmentally benign and easily leads to the synthesis of desired product i.e. ethyl 4-phenyl-2,7,7-trimethyl-5-oxo-1,4,5,6,7,8hexahydroquinoline-3-carboxylate. The catalyst is easily available and inexpensive. This method proves to be advantageous in terms of excellent yields (80-90\%) and short reaction times (30-60 min).
\end{abstract}

Keywords: Hantzsch reaction, Green chemistry, TEBAC, Polyhydroquinolines

\section{Introduction}

Nowadays, researchers are dynamically taking on the challenge of developing green synthetic methodologies to meet the criteria of green, sustainable, environmentally conscious chemistry ${ }^{1,2}$. Recently, practical procedures in the absence of solvents and catalysts have been accomplished for greener and cleaner syntheses ${ }^{3}$. These aspects have inspired both the synthetic and mechanistic chemists all over the world to work on developing new synthetic methodologies based on green chemistry. Polyhydroquinolines containing 1,4-dihydropyridine moiety possess a variety of biological activities such as vasodilator, bronchodilator, antiatherosclerotic, antitumor, geroprotective, hepatoprotective and antidiabetic activities ${ }^{4,5}$. These compounds exhibit several other medicinal applications including neuroprotectant and platet anti-aggregator activity in addition to acting as cerebral antiischemic agents in the treatment of Alzheimer's disease ${ }^{5}$.

Owing to such significance, the synthesis of substituted polyhydroquinolines has been a subject of great focus in organic chemistry. Numerous methods have been reported such as conventional heating ${ }^{6}$ as well as presence of various catalysts such as $L$-proline ${ }^{7}$, ceric ammonium nitrate $(\mathrm{CAN})^{8}$, silica-perchloric acid $\left(\mathrm{HClO} 4-\mathrm{SiO}_{2}\right)^{9}$, nickel nanoparticle ${ }^{10}$, $\mathrm{FeF}_{3}{ }^{11}, \mathrm{~K} 7\left[\mathrm{PW} 11 \mathrm{CoO}_{40}\right]^{12}$, solar heat $^{13}$, bakers' yeast ${ }^{14}, \mathrm{Yb}(\mathrm{OTf}) 3^{15}, \mathrm{Sc}(\mathrm{OTf}) 3^{16}$ and $\mathrm{HClO} 4-\mathrm{SiO}_{2}{ }^{17}$. However, these methods suffer from several disadvantages such as moisture sensitive, highly toxic, unpleasant experimental procedure, expensive reagents, use of larger 
quantities of volatile organic solvents, long reaction times, low yields and harsh reaction conditions $^{18}$. Therefore, it is necessary to develop an efficient and versatile method for the synthesis of polyhydroquinoline derivatives which tends to initiate the search for a better catalyst using less hazardous solvents or solvent free conditions. The triethylbenzylammoniumchloride (TEBAC) catalyst used in present work is an efficient and reusable catalyst and in its presence different derivatives of ethyl- 4-phenyl-2,7,7-trimethyl-5-oxo1,4,5,6,7,8-hexahydroquinoline-3-carboxylate are successfully synthesized and characterized.

\section{Experimental}

Chemicals were purchased from Merck, Fluka and Aldrich chemical companies. All the products were identified by comparison of their physical and spectral data with those of the authentic samples. Melting points were determined using an electrothermal apparatus and are uncorrected. The progress of the reaction was monitored by thin layer chromatography (TLC) using silica gel plates. IR spectra (KBr disc) were recorded on a FTIR instrument and ${ }^{1} \mathrm{H}$ NMR $\left(\mathrm{CDCl}_{3}\right)$ ( $\delta \mathrm{ppm}$ downfield from TMS) spectra were obtained on Bruker 400 ultrasheild $(400 \mathrm{MHz})$ spectrometer. Homogeneity of compounds was routinely checked on silica gel-G TLC plates using benzene: ethylacetate(9:1) as eluent.

\section{General procedure for preparation of polyhydroquinolines}

A mixture of arylaldehyde $(1 \mathrm{mmol})$, ethylacetoacetate $(1 \mathrm{mmol})$, dimedone $(1 \mathrm{mmol})$ and ammonium acetate $(1.5 \mathrm{mmol})$ in the presence of TEBAC $(150 \mathrm{mg})$ was stirred at ambient temperature for a particular period of time. The progress of the reaction was monitored by TLC. After completion of the reaction, the reaction mixture was washed with water and pure product was obtained after recrystalization with ethylacetate in $80-90 \%$ yield.

\section{Results and Discussion}

As a part of continuing efforts toward the development of green synthesis, method for synthesis of different derivatives of ethyl-4-phenyl-2,7,7-trimethyl-5-oxo-1,4,5,6,7,8hexahydroquinoline- 3-carboxylates using dimedone, ethylacetoacetate, ammonium acetate and arylaldehydes in 1:1:1.5:1 ratio under solvent free conditions is as shown in Scheme 1.

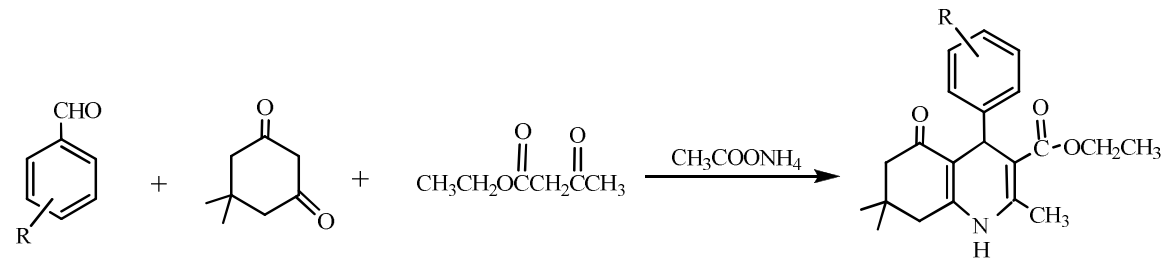

\section{Scheme 1}

The reactions were completed within 30-60 minutes at room temperature and the products were obtained via filtration and recrystallization by ethylacetate. In this, the reaction mixtures were stirred on a magnetic stirrer and progress of the reactions monitored with the help of TLC. The best results were obtained by using optimum amount (150 mg) of catalyst (TEBAC) at room temperature and solvent free reaction conditions. The various arylaldehydes used were 4-chlorobenzaldehyde (1a) 2,4-dichlorobenzaldehyde (1b), 4methylbenzaldehyde (1c), 4-methoxybenzaldehyde (1d), 3,4-dimethoxybenzaldehyde (1e), 4-nitrobenzaldehyde (1f) and 4-bromobenzaldehyde (1g). In all the cases, using the optimized reaction conditions, the reactions proceeded smoothly to furnish the desired polyhydroquinoline derivatives in $80-90 \%$ yields. 


\section{Effect of catalyst concentration}

Optimum amount of catalyst was investigated from the reference reaction in which 4-chlorobenzaldehyde chosen as a representative aldehyde was reacted with dimedone, ethylacetoacetate and ammonium acetate. The amount of catalyst was varied from $50 \mathrm{mg}$ to $200 \mathrm{mg}$ to observe the effect on the yield of product. Table 1 shows the effect of catalyst concentration on the yield of the corresponding polyhydroquinoline derivative. It has been found that yield of product increased with increasing catalyst concentration from 50 to $150 \mathrm{mg}$. Further addition of catalyst had no noticeable effect on the yield but rather yield was decreased. Thus, in all other reactions an amount of $150 \mathrm{mg}$ of TEBAC was used.

Table 1. Catalyst effect on the synthesis of ethyl-4-phenyl-2,7,7-trimethyl-5-oxo1,4,5,6,7,8-hexahydroquinoline-3-carboxylate.

\begin{tabular}{cccc}
\hline Entry & Amount of TEBAC, mg & Reaction time, min & Yield, \% \\
\hline 1 & 50 & 60 & 65 \\
2 & 100 & 60 & 70 \\
3 & 150 & 60 & 90 \\
4 & 200 & 60 & 85 \\
\hline
\end{tabular}

Synthesis of ethyl-4-phenyl-2,7,7-trimethyl-5-oxo-1,4,5,6,7,8-hexahydroquinoline-3carboxylate

The results of the reactions of aryl aldehydes, ethyl acetoacetate, dimedone and ammonium acetate in the presence of TEBAC at room temperature are shown in Table 2. Various substituted aromatic aldehydes reacted well to yield the corresponding ethyl- 4-phenyl-2,7,7trimethyl-5-oxo-1,4,5,6,7,8-hexahydroquinoline-3-carboxylates.

Table 2. Results of synthesis of ethyl-4-phenyl-2,7,7-trimethyl-5-oxo-1,4,5,6,7,8hexahydroquinoline-3-carboxylates in the presence of TEBAC as per Scheme 1

\begin{tabular}{|c|c|c|c|}
\hline$\underset{\Xi}{\vec{U}} \vec{\simeq}$ & Product & 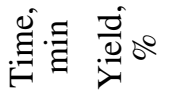 & m.p $\left({ }^{\circ} \mathrm{C}\right)$ Obs. [Lit.] \\
\hline $1 \mathbf{1}(\mathbf{a})$ & $\begin{array}{l}\text { Ethyl-4-(4-chlorophenyl)-2,7,7- } \\
\text { trimethyl-5-oxo-1,4,5,6,7,8- } \\
\text { hexahydroquinoline-3-carboxylate (2a) }\end{array}$ & 85 & $244-246{ }^{\circ} \mathrm{C}\left[245-247^{\circ} \mathrm{C}\right]^{14}$ \\
\hline 2 1(b) & $\begin{array}{l}\text { Ethyl-4-(2,4-dichlorophenyl)-2,7,7- } \\
\text { trimethyl-5-oxo-1,4,5,6,7,8- } \\
\text { hexahydroquinoline-3-carboxylate }(\mathbf{2 b})\end{array}$ & 90 & $280-282^{\circ} \mathrm{C}\left[281-283^{\circ} \mathrm{C}\right]^{14}$ \\
\hline $31(\mathbf{c})$ & $\begin{array}{l}\text { Ethyl-4-(4-methylphenyl)-2,7,7- } \\
\text { trimethyl-5-oxo-1,4,5,6,7,8- } \\
\text { hexahydroquinoline-3-carboxylate } \quad(\mathbf{2 c})\end{array}$ & 55 & $224-226^{\circ} \mathrm{C}\left[226-228^{\circ} \mathrm{C}\right]^{9}$ \\
\hline $4 \mathbf{1}(\mathbf{d})$ & $\begin{array}{l}\text { Ethyl-4-(4-methoxyphenyl)-2,7,7- } \\
\text { trimethyl-5-oxo-1,4,5,6,7,8- } \\
\text { hexahydroquinoline-3-carboxylate (2d) }\end{array}$ & 85 & $258-260^{\circ} \mathrm{C}\left[259-261^{\circ} \mathrm{C}\right]^{11}$ \\
\hline $5 \quad 1(e)$ & $\begin{array}{l}\text { Ethyl-4-(3,4-dimethoxyphenyl)-2,7,7- } \\
\text { trimethyl-5-oxo-1,4,5,6,7,8- } \\
\text { hexahydroquinoline-3-carboxylate (2e) }\end{array}$ & 60 & $286-288^{\circ} \mathrm{C}\left[287-289^{\circ} \mathrm{C}\right]^{14}$ \\
\hline $6 \mathbf{1 ( f )}$ & $\begin{array}{l}\text { Ethyl-4-(4-nitrophenyl)-2,7,7-trimethyl- } \\
\text { 5-oxo-1,4,5,6,7,8-hexahydroquinoline-3- } \\
\text { carboxylate (2f) }\end{array}$ & 45 & $240-242^{\circ} \mathrm{C}\left[241-243^{\circ} \mathrm{C}\right]^{I \mathrm{I}}$ \\
\hline 7 1(g) & $\begin{array}{l}\text { Ethyl-4-(4-bromophenyl)-2,7,7- } \\
\text { trimethyl-5-oxo-1,4,5,6,7,8- } \\
\text { hexahydroquinoline-3-carboxylate (2g) }\end{array}$ & 60 & $242-244^{\circ} \mathrm{C}\left[244-246^{\circ} \mathrm{C}\right]^{11}$ \\
\hline
\end{tabular}




\section{The physical and spectroscopic data of compounds}

Ethyl-4-(4-chlorophenyl)-2,7,7-trimethyl-5-oxo-1,4,5,6,7,8-hexahydroquinoline-3carboxylate (1a)

M.p.244-246 ${ }^{\circ} \mathrm{C} ; \operatorname{IR}\left(\mathrm{cm}^{-1}\right)$ : 3346, 3054, 1700; ${ }^{1} \mathrm{H} \mathrm{NMR}\left(\mathrm{CDCl}_{3}\right): 0.85$ (s, 3H), $1.05\left(\mathrm{~s}, \mathrm{CH}_{3}\right)$, $1.20(\mathrm{t}, 3 \mathrm{H}), 2.30(\mathrm{~m}, 4 \mathrm{H}), 2.35(\mathrm{~s}, 3 \mathrm{H}), 3.75(\mathrm{q}, 4 \mathrm{H}), 5.30(\mathrm{~s}, \mathrm{CH}), 5.80(\mathrm{br}, \mathrm{NH}), 6.70-7.10$ (m, ArH),

Ethyl-4-(2,4-dichlorophenyl)-2,7,7-trimethyl-5-oxo-1,4,5,6,7,8-hexahydroquinoline3-carboxylate (1b)

M.p.280-282 ${ }^{\circ} \mathrm{C}$; IR( $\left(\mathrm{cm}^{-1}\right): 3374,3034,1690 ;{ }^{1} \mathrm{H} \mathrm{NMR}\left(\mathrm{CDCl}_{3}\right): 0.90$ (s, 3H), $1.10\left(\mathrm{~s}, \mathrm{CH}_{3}\right)$, $1.30(\mathrm{t}, 3 \mathrm{H}), 2.50(\mathrm{~m}, 4 \mathrm{H}), 2.80(\mathrm{~s}, 3 \mathrm{H}), 3.85(\mathrm{q}, 4 \mathrm{H}), 5.54(\mathrm{~s}, \mathrm{CH}), 5.89(\mathrm{br}, \mathrm{NH}), 6.75-7.10$ $(\mathrm{m}, \mathrm{ArH})$.

Ethyl-4-(4-methylphenyl)-2,7,7-trimethyl-5-oxo-1,4,5,6,7,8-hexahydroquinoline-3carboxylate (1c)

M.p.224-226 ${ }^{\circ} \mathrm{C} ; \operatorname{IR}\left(\mathrm{cm}^{-1}\right): 3306,2994,1690 ;{ }^{1} \mathrm{H} \mathrm{NMR}\left(\mathrm{CDCl}_{3}\right): 0.89$ (s, 3H), 1.00 (s, 3H), $1.25(\mathrm{t}, 3 \mathrm{H}), 2.35(\mathrm{~m}, 4 \mathrm{H}), 2.60(\mathrm{~s}, 3 \mathrm{H}), 3.50(\mathrm{~s}, 3 \mathrm{H}), 4.00(\mathrm{q}, 4 \mathrm{H}), 5.25(\mathrm{~s}, \mathrm{CH}), 5.80(\mathrm{br}$, $\mathrm{NH})$, 7.00-7.20 (m, ArH).

Ethyl-4-(4-methoxyphenyl)-2,7,7-trimethyl-5-oxo-1,4,5,6,7,8-hexahydroquinoline-3carboxylate (1d)

M.p.250-260 ${ }^{\circ} \mathrm{C} ; \operatorname{IR}\left(\mathrm{cm}^{-1}\right): 3314,3005,1670 ;{ }^{1} \mathrm{H} \mathrm{NMR}\left(\mathrm{CDCl}_{3}\right): 0.85$ (s, 3H), 1.00 (s, 3H), $1.15(\mathrm{t}, 3 \mathrm{H}), 2.35(\mathrm{~m}, 4 \mathrm{H}), 2.50(\mathrm{~s}, 3 \mathrm{H}), 3.30(\mathrm{~s}, 3 \mathrm{H}), 4.00(\mathrm{q}, 4 \mathrm{H}), 4.75(\mathrm{~s}, \mathrm{CH}), 5.60$ (br, $\mathrm{NH})$, 7.00-7.15 (m, ArH).

Ethyl-4-(3,4-dimethoxyphenyl)-2,7,7-trimethyl-5-oxo-1,4,5,6,7,8-hexahydro-quinoline3-carboxylate (1e)

M.p.286-298 ${ }^{\circ} \mathrm{C} ; \operatorname{IR}\left(\mathrm{cm}^{-1}\right): 3274,2997,1687 ;{ }^{1} \mathrm{H} \mathrm{NMR}\left(\mathrm{CDCl}_{3}\right): 0.95$ (s, 6H), 1.05 (s, 3H), $1.30(\mathrm{t}, 3 \mathrm{H}), 2.35(\mathrm{~m}, 4 \mathrm{H}), 2.55(\mathrm{~s}, 3 \mathrm{H}), 3.60(\mathrm{~s}, 3 \mathrm{H}), 3.65(\mathrm{~s}, 3 \mathrm{H}), 4.15(\mathrm{q}, 2 \mathrm{H}), 5.05(\mathrm{~s}, \mathrm{CH})$, 5.70 (br, NH), 6.75-7.10 (m, ArH).

Ethyl-4-(4-nitrophenyl)-2,7,7-trimethyl-5-oxo-1,4,5,6,7,8-hexahydroquinoline-3carboxylate (1f)

M.p.240-242 ${ }^{\circ} \mathrm{C} ; \operatorname{IR}\left(\mathrm{cm}^{-1}\right): 3324,3012,1696 ;{ }^{1} \mathrm{H} \mathrm{NMR}\left(\mathrm{CDCl}_{3}\right): 0.90$ (s, 3H), 1.05 (s, 3H), $1.25(\mathrm{t}, 3 \mathrm{H}), 2.30\left(\mathrm{~m}, \mathrm{CH}_{2}, 4 \mathrm{H}\right), 2.40(\mathrm{~s}, 3 \mathrm{H}), 3.85-3.90(\mathrm{q}, 4 \mathrm{H}), 5.10(\mathrm{~s}, \mathrm{CH}), 5.65(\mathrm{~s}, \mathrm{NH})$, 6.95-7.20 (m, ArH).

Ethyl-4-(4-bromophenyl)-2,7,7-trimethyl-5-oxo-1,4,5,6,7,8-hexahydro-quinoline-3carboxylate (1g)

M.p.242-244 ${ }^{\circ} \mathrm{C} ; \operatorname{IR}\left(\mathrm{cm}^{-1}\right): 3374,3034,1699 ;{ }^{1} \mathrm{H} \mathrm{NMR}\left(\mathrm{CDCl}_{3}\right): 0.95$ (s, 6H), 1.10 (s, 3H), $1.21(\mathrm{t}, 3 \mathrm{H}), 2.40\left(\mathrm{~m}, \mathrm{CH}_{2}, 4 \mathrm{H}\right), 2.60(\mathrm{~s}, 3 \mathrm{H}), 4.00(\mathrm{q}, 4 \mathrm{H}), 5.20(\mathrm{~s}, \mathrm{CH}), 5.85(\mathrm{~s}, \mathrm{NH}), 7.00-$ $7.20(\mathrm{~m}, \mathrm{ArH})$.

\section{Conclusion}

A simple, efficient and economical method has been described for the synthesis of polyhydroquinolines. This environmentally benign and safe procedure is advantageous in terms of mild experimental conditions, economic catalyst, excellent yields, and short reaction times, easy isolation of products and absence of any organic solvent and large scale applicability. 


\section{Acknowledgment}

The authors are thankful to the Head, Department of Chemistry, Maharshi Dayanand University, Rohtak for providing facilities for doing research work.

\section{References}

1. István T and Paul T, Chem Rev., 2007, 107(6), 2167-2168; DOI:10.1021/cr0783784

2. $\quad$ Ranu B C, Dey S S and Hajra A, Green Chem., 2003, 5, 44-46; DOI:10.1039/B211238H

3. Halimehjani A Z, Pourshojaei Y and Saidi M R, Tetrahedron Lett., 2009, 50(1), 32-34; DOI:10.1016/j.tetlet.2008.10.063

4. Sainani J B, Shah A C and Arya V P, Indian J Chem B, 1994, 33, 526-531.

5. Paidepala H, Nageudra S, Saddanappu V, Addlagatta A and Das B, Med Chem Res., 2014, 23(2), 1031-1036; DOI:10.1007/s00044-013-0706-1

6. Bretzel R G, Bollen C C, Maeser E and Fererlin K F, Drugs Futur., 1992, 17, 465-468.

7. Sufirez M, Ochoa E, Verdecia Y, Beatriz Pita, LourdesMorán, Nazario Martín, Margarita Quinteiro, Carlos Seoane, José L Soto, Hector Novoa, Norbert Blaton and Oswald M Peters, Tetrahedron, 1999, 55(3), 875-884; DOI:10.1016/S00404020(98)01078-3

8. Karade N N, Budhewar V H, Shinde S V and Jadhav W N, Lett Org Chem., 2007, 4(1), 16-19; DOI:10.2174/157017807780037405

9. Maheswara M, Siddaiah V, Damu G L V and Rao C V, Arkivoc, 2006, 2, 201-206.

10. Sapkal S B, Shelke K F, Shingate B B and Shingare M S, Tetrahedron Lett., 2009, 50(15), 1754-1756; DOI:10.1016/j.tetlet.2009.01.140

11. Surasani R, Kalita D, Rao A V D, Yarbagi K and Chandrasekhar K B, J Fluorine Chem., 2012, 135, 91-96; DOI:10.1016/j.jfluchem.2011.09.005

12. Heravi M M, Bakhtiari K, Javadi N M, Bamoharram F F, Saeedi M and Oskooie H A, J Mole Catal A: Chem., 2007, 264(1-2), 50-52; DOI:10.1016/j.molcata.2006.09.004

13. Mekheimer R A, Hameed A A and Sadek K U, Green Chem., 2008, 10(5), 592-593; DOI:10.1039/B715126H

14. Kumar A and Maurya R A, Tetrahedron Lett., 2007, 48(22), 3887-3890; DOI:10.1016/j.tetlet.2007.03.130

15. Kidwai M, Chauhan R, Bhatnagar D, Singh A K, Mishra B and Dey S, Monatshefte fur Chemie, 2012, 143(12), 1675-1680; DOI:10.1007\%2Fs00706-012-0742-4

16. Wang L M, Sheng J, Zhang L, Han J W, Fan Z, Tian H and Qian C T, Tetrahedron, 2005, 61, 1539-1543; DOI:10.1016/j.tet.2004.11.079

17. Donelson J L, Gibbs R A and De S K, J Mole Catal A: Chem., 2006, 25(1-2), 309312; DOI:10.1016/j.molcata.2006.09.035

18. Kumar A and Maurya R A, Tetrahedron, 2007, 63(9), 1946-1952;

DOI:10.1016/j.tet.2006.12.074 